\title{
Interactions and magnetic viscosity: Nonmonotonic time variation of the magnetization during relaxation at constant demagnetizing field
}

\author{
C. de Julián, M. Emura, ${ }^{\text {a) }}$ F. Cebollada, and J. M. González ${ }^{\text {b) }}$ \\ Departamento de Propiedades Ópticas, Magnéticas y de Transporte, Instituto de Ciencia de Materiales de \\ Madrid-Consejo Superior de Investigaciones Científicas, Cantoblanco, 28049 Madrid, Spain
}

(Received 22 July 1996; accepted for publication 25 October 1996)

\begin{abstract}
We present magnetic relaxation data of PrNdFeB magnets. These data were obtained by measuring the time evolution of the magnetization under constant applied demagnetizing fields. The results corresponding to fields far from the range of the coercive force evidenced a nonmonotonic time variation of the magnetization. We propose that the occurrence of magnetic interactions underlies the observed anomalous behavior. This idea is checked through a micromagnetic simulation of the time evolution of the magnetization of a low field reversed nucleus which is exchange and magnetostically coupled to the main hard phase. (C) 1996 American Institute of Physics.
\end{abstract} [S0003-6951(96)01053-4]

The phenomenology of magnetic relaxation is commonly discussed by accepting that the studied samples can be described in terms of a set of local, noninteracting subsystems, demagnetizing through a simple mechanism and fully describable in terms of remanence and reversed states. ${ }^{1}$ The kinetics for this relaxation process is considered to be the Arrhenius-Néel one, ${ }^{2}$ which predicts a monotonic decrease of the component of the magnetization measured along the applied field direction. The validity of the above mentioned hypotheses is not clear since many experimental evidences indicate that the reversal processes should be described in terms of a sequence of events involving nucleation, nucleus expansion, and/or domain wall pinningdepinning processes as elemental stages. ${ }^{3-5}$ Also, it is widely recognized as the relevant role of the exchange and dipolar interactions both in hysteresis ${ }^{4,6}$ and in relaxation. Finally, the occurrence of non-Arrhenius behavior has been evidenced both in isolated particle experiments ${ }^{7}$ and in micromagnetic simulations. ${ }^{8}$ A further evidence against the simple two-level description of the relaxation is the occurrence of negative viscosity coefficients both in minor ${ }^{9,10}$ and in mayor loops. ${ }^{11}$ This seems to be a quite general effect since it has been observed both in single phase ${ }^{11}$ and in composite ${ }^{9}$ hard materials. The phenomenology has been associated, in two phase systems, to the exchange coupling, ${ }^{9}$ whereas in Ref. 10 it was evidenced that this type of coupling is not a prerequisite for the observation of that anomalous behavior. Trying to contribute to the clarification of the origin of the negative viscosity and to the discussion on the classic treatment of the relaxation phenomena, we will present here viscosity results corresponding to relaxation fields far from the coercive force. Those results will be discussed on the basis of a micromagnetic simulation. We have studied a $\operatorname{Pr}_{6} \mathrm{Nd}_{9} \mathrm{Fe}_{76} \mathrm{~B}_{9}$ sample which was prepared by crystallizing (continuous heating at $40 \mathrm{~K} \mathrm{~min}^{-1}$ up to $973 \mathrm{~K}$ ) a melt spun amorphous precursor. X-ray diffractograms taken in the crystallized material revealed a majority presence of a 2:14:1 phase and minority $\alpha$-Fe reflections. The magnetic measurements were

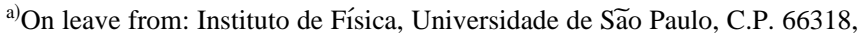
05389-970, São Paulo, SP, Brazil.

${ }^{b)}$ Electronic mail: immg4a@fresno.csic.es
}

carried out using a superconducting quantum interference device (SQUID) magnetometer and applying the field along the casting direction of the flake-shaped samples. At all the considered temperatures, the measured demagnetization branch of the hysteresis loop of the sample revealed the occurrence of a rapid magnetization decrease near the remanence, evidencing that, in contrast to the samples measured in Ref. 9, the soft and hard phases were exchange decoupled to a large extent. The viscosity experiments were carried out by initially submitting the sample to a positive $5 \mathrm{~T}$ field and then decreasing it, at a constant rate of $0.05 \mathrm{~T} \mathrm{~min}^{-1}$, down to a value in the second quadrant which was kept constant during the acquisition of the magnetization data. In Fig. 1, we compare the relaxation behavior of our sample (measured at 175 $\mathrm{K})$ for three different fields: below $(2 \mathrm{~T})$, above $(4.5 \mathrm{~T})$, and at the critical field value $(3.7 \mathrm{~T})$. From that figure, it is apparent that the time variation of the magnetization, $\mu_{0} M_{z}(t)$, is monotonic when the measuring field is in the range at which most of the irreversibilities occur. In clear contrast with this, the relaxation at fields far from the coercive force shows a nonmonotonic behavior. In order to understand this anomalous behavior, let us consider that our sample can be represented by a collection of interacting twolevel subsystems. We will restrict ourselves to the case in which the applied field is smaller than the critical one, but

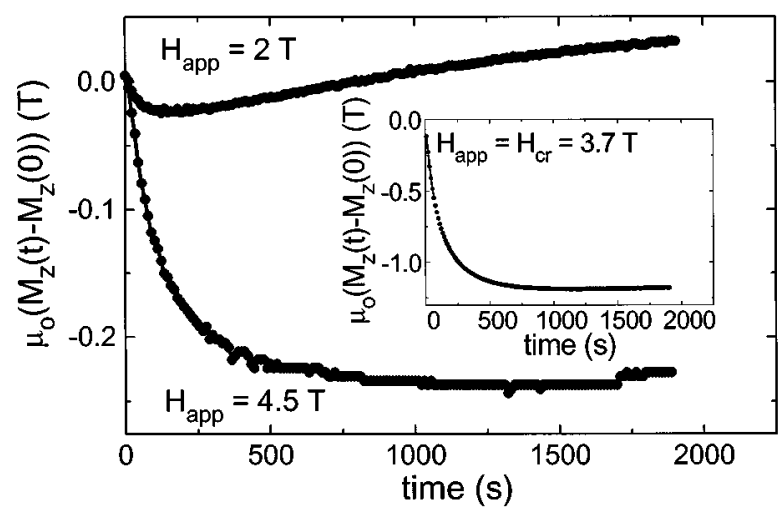

FIG. 1. Time dependence of the magnetization for fields below and above the critical one (data obtained at $175 \mathrm{~K}$ ). The inset shows the dependence of the magnetization at the critical field. 
our argument can be easily translated to a field value above the critical field. In that situation, the local magnetization reversal is possible if, at one of the subsystems forming the sample, the barrier protecting the local remanence is, due to a reduced local anisotropy value, small enough to be overcome either because the applied field is sufficient to induce the reversal or because the thermal energy is of the order of the barrier height. If, due to a reduced gradient of the local properties and to a non-negligible exchange coupling, the limiting wall of the reversed region can easily propagate to a larger region of the sample (for instance, to the entire grain containing the initially reversed region), it could be possible for the system to reach a configuration of larger energy than that corresponding to the initial unreversed state. To give an example of that possibility, let us mention the increase in dipolar energy that should be associated with the presence of poles at the frontier between the reversed and unreversed regions. Once at this higher energy state (and assuming some degree of adiabaticity), the system could start to relax again. A possible mechanism for that relaxation (which should get rid of the excess of dipolar energy by lowering the pole density at the reversed region interface) is the formation of an extended inhomogeneous magnetic moment structure (similar to closure domains) which could increase the component of the magnetization along the initial saturation direction. In order to consider this idea in further detail, we have carried out a micromagnetic simulation of the behavior of a system in which a reversed nucleus is formed under small applied fields. Our model consisted of a square $(24 \times 24)$ array of infinite atomic lines (parallel to the $X$ axis of our reference system). Intraline exchange interactions are considered to be infinite, whereas the corresponding interline couplings are finite. The intrinsic magnetic properties are defined for each line by (i) the exchange energy-to-anisotropy energy ratio, $a \equiv A d^{-2} / K=2$, where $A, d=2.5 \times 10^{-10} \mathrm{~m}$ and $K=10^{7} \mathrm{Jm}^{-3}$ are the exchange constant, the interatomic distance, and the anisotropy constant of the hard phase, respectively; (ii) the dipolar energy-to-anisotropy energy ratio, $m \equiv \mu_{0} M_{S}^{2} / 2 K=0.3$, where $\mu_{0} M_{S}$ is the saturation magnetization and (iii) the local easy-axis orientation (for all the atomic lines in the system, we have considered that the local easy axis is contained in the $Y Z$ plane and tilted $20^{\circ}$ away from the $Z$ axis). The internal energy of our system includes uniaxial anisotropy, Zeeman (field applied along the $-Z$ axis), exchange (first neighbors), and dipolar terms. The energy evolution is followed by a Monte Carlo algorithm. We considered that our system is kept at constant temperature (corresponding to $10^{-4}$ of the maximum anisotropy energy per unit length attainable by the system). We define a Monte Carlo step (MCS) as the process corresponding to the introduction of random modifications in all the degrees of freedom of the system. If the attempt frequency for such modifications is known, then the number of MCS can be converted into time units. ${ }^{12}$ For the sake of simplification, the simulated relaxation process starts from a homogeneous magnetization configuration, upon the artificial introduction of a reversed nucleus (a very similar starting point can be achieved by associating a reduced anisotropy to a region of the model system). Considering the large degree of decoupling of the two phases forming the sample which is sug-

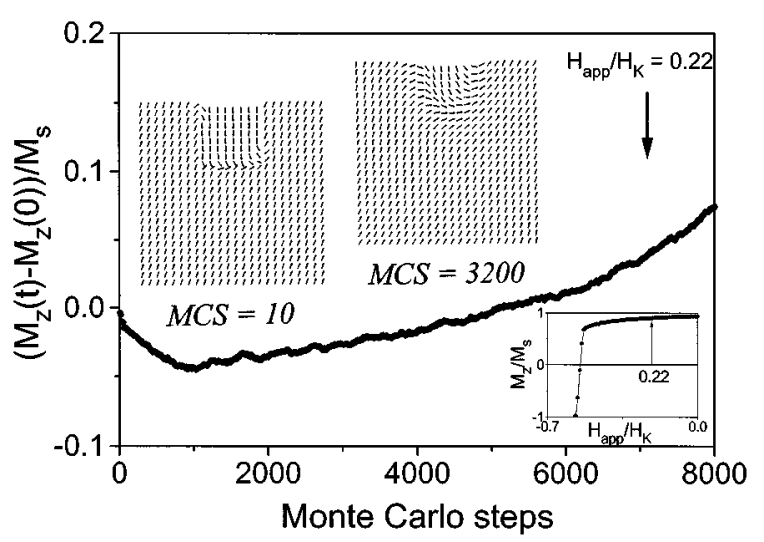

FIG. 2. Time $(\mathrm{MCS})$ evolution of the reduced magnetization of a $(24 \times 24)$ model system in which a $(8 \times 8)$ reversed nucleus was present. The insets show the configuration of the system for 10 and $3200 \mathrm{MCS}$, and the reduced (to the anisotropy field, $H_{K}$ ) applied field, $H_{\text {app }}$, dependence of the reduced (to the saturation value, $M_{S}$ ) $Z$ component of the magnetization.

gested by the hysteretic behavior, that reversed nucleus could be identified with a small anisotropy region inside a hard grain. The time variation of the magnetization data presented in Fig. 2 (evaluated for an applied field value much smaller than the critical one, see the inset in Fig. 2) corresponds to a simulation of the relaxation of a system in whose surface a $(8 \times 8)$ reversed nucleus was present. As is clear from Fig. 2 our simulation qualitatively reproduces the nonmonotonic time variation of the magnetization. It is interesting to note that the artificially reversed nucleus is considerably stable: despite the reduced applied field value, the exchange interactions of the moments on its border with those immediately out from it, are not sufficient to induce either a global reversal or a rapid remagnetization. What, in fact, happens (see the inset in Fig. 2, where the configuration of the system for two MCS values is shown) is that an extended domain walltype structure forms at the border of the nucleus, which results in a decrease of the magnetization component along the field direction (the relationship of this process with the exchange coupling is evidenced by the rapid evolution of the corresponding energy term during the MCS range associated to magnetization decrease). After ca. $10^{3} \mathrm{MCS}$, the nucleus starts getting more inhomogeneous and shrinking slowly, which leads to an increase of that component (the only energy contributions significantly evolving during this process are the dipolar and the Zeeman ones). In contrast to the results in Fig. 2, the simulation of the relaxation process of the same system under fields of the order of the critical one showed that a global reversal followed, very rapidly, the introduction of a reversed nucleus (this result was independent of the nucleus size and position).

In summary, we have experimentally observed a nonmonotonic time variation of the magnetization when that quantity is measured under constant fields far from the critical field range. Our results were discussed in terms of the occurrence of interactions between a nucleus which gets reversed for fields clearly different from those corresponding to the reversal of its neighboring regions and which is coupled to them through exchange and dipolar interactions. From our results, we conclude that the exchange interactions 
are on the origin of the initial expansion of that reversed nucleus, whereas the dipolar coupling underlies its further decrease in size.

${ }^{1}$ R. Street, R. K. Day, and J. B. Dunlop, J. Magn. Magn. Mater. 69, 106 (1987).

${ }^{2}$ L. Néel, Adv. Phys. 4, 191 (1955).

${ }^{3}$ D. W. Taylor, V. Villas-Boas, Q. Lu, M. F. Rossignol, F. P. Missell, D. Givord, and S. Hirosawa, J. Magn. Magn. Mater. 130, 225 (1994).

${ }^{4}$ A. Hernando, I. Navarro, and J. M. González, Europhys. Lett. 20, 175 (1992).

${ }^{5}$ G. C. Hadjipanayis, in Science and Technology of Nanostructured Mag- netic Materials, edited by G. C. Hadjipanayis and G. A. Prinz (Plenum, New York, 1991), p. 607.

${ }^{6}$ R. Coehoorn and C. de Waard, J. Magn. Magn. Mater. 83, 228 (1990).

${ }^{7}$ M. Lederman, S. Schultz, and M. Ozaki, Phys. Rev. Lett. 73, 1986 (1994).

${ }^{8}$ J. M. González, R. Ramírez, R. Smirnov-Rueda, and J. González, Phys. Rev. B 52, 16034 (1995).

${ }^{9}$ E. H. Feutrill, L. Folks, P. G. McCormick, P. A. I. Smith, and R. Street, Proceedings of the 8th International Symposium on Magnetic Anisotropy and Coercitivity in RE-TM alloys (Birmingham, 1994), p. 297.

${ }^{10}$ K. O'Grady and S. J. Greaves, J. Magn. Magn. Mater. 138, L233 (1994).

${ }^{11}$ K.-H. Müller, D. Eckart, A. Handstein, M. Wolf, S. Collocott, and C. Andrikidis, Proceedings of the 9th International Symposium on Magnetic Anisotropy and Coercivity in RE-TM alloys (São Paulo, 1996), p. 381.

${ }^{12}$ D. W. Heermann, in Computer Simulation Methods in Theoretical Physics (Springer, Berlin, 1986), p. 78. 